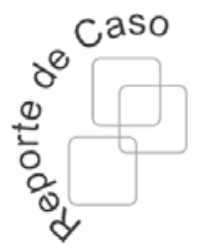

Citación: Contreras L. y Granados W., "Diseño de un dispositivo para la movilidad de personas con discapacidad motriz usando el método función de calidad," Ingeniería, vol. 19, no. 1, pp. 65-82, 2014

\title{
Diseño de un dispositivo para la movilidad de personas con discapacidad motriz usando el método función de calidad
}

\author{
Design of a Mobility Device for Disabled \\ People Using the Method of Quality \\ Function Deployment
}

Leonardo Emiro

Contreras Bravo

Universidad Distrital

Francisco José de Caldas

lecontrerasb@udistrital.edu.co

Wilmar Augusto Granados Universidad Distrital

Francisco José de Caldas wagranados@udistrital.edu.co

\section{( $) \odot$}

Fecha recibido: $\quad 07 / 09 / 2013$ Fecha modificado: 11/06/2013 Fecha aceptado: 16/06/2014

\section{Resumen}

Este artículo describe el diseño de un dispositivo de movilidad para personas con discapacidad motriz entre 8 y 15 años de edad, con patologías T12 y que no tengan patologías concomitantes en miembros superiores. Inicia con la obtención de los requerimientos del cliente, y la transformación de estos a partir de la herramienta llamada función de calidad (QFD - Quality Function Deployment), y el uso de diferentes métodos de evaluación del diseño obtenido.

Después de caracterizar de una manera cuantitativa lo cualitativo, se procede a desarrollar un proceso sistémico que genere elementos particulares para llegar a concretar el diseño que une los elementos técnicos y los requisitos del cliente (usuario). En la parte final se realizan varios diseños mediante el software Solid Edge. Se selecciona un modelo que cumple con los requisitos del cliente arrojados por la herramienta QFD y otras metodologías de diseño

Palabras clave: función de calidad, discapacidad, ingeniería, movilidad, QFD.

\section{Abstract}

This paper describes the design of a mobility device for physically disabled people between 8-15 years with T12 pathologies and no co-morbidities in the upper limbs. It begins by eliciting the customer's requirements, and conversion to designs prototypes using a tool called quality function (QFD - Quality Function Deployment). The evaluation of the different designs is also shown. 
Once the qualitative description is characterized quantitatively, the design process proceeds by systemically generating particular elements to reach the global design by joining the technical elements and requirements of the user. In the final part of the paper, several designs using Solid Edge software are presented. Lastly, the model that better meets customer requirements indicated by the QFD analysis is chosen.

Key words: disability, engineering, mobility, QFD, quality.

\section{Introducción}

Según [1], en Colombia conviven alrededor de 4992000 discapacitados que equivaldrían a $12 \%$ de la población. Por otro lado, según el Censo 2005 (dato más reciente en el país) existen alrededor de 6.3 personas por cada 100 con una discapacidad permanente, en donde parte de esa población discapacitada tiene entre 8 y 13 años. En la figura 1 se muestra que la población proyectada para el 2013, a partir del Censo 2005, para personas de sexo masculino entre 10 y 15 años es alrededor de 16000 discapacitados, mientras que para el sexo femenino del mismo rango de edad es un tanto menor. Datos que van en aumento según la proyección.

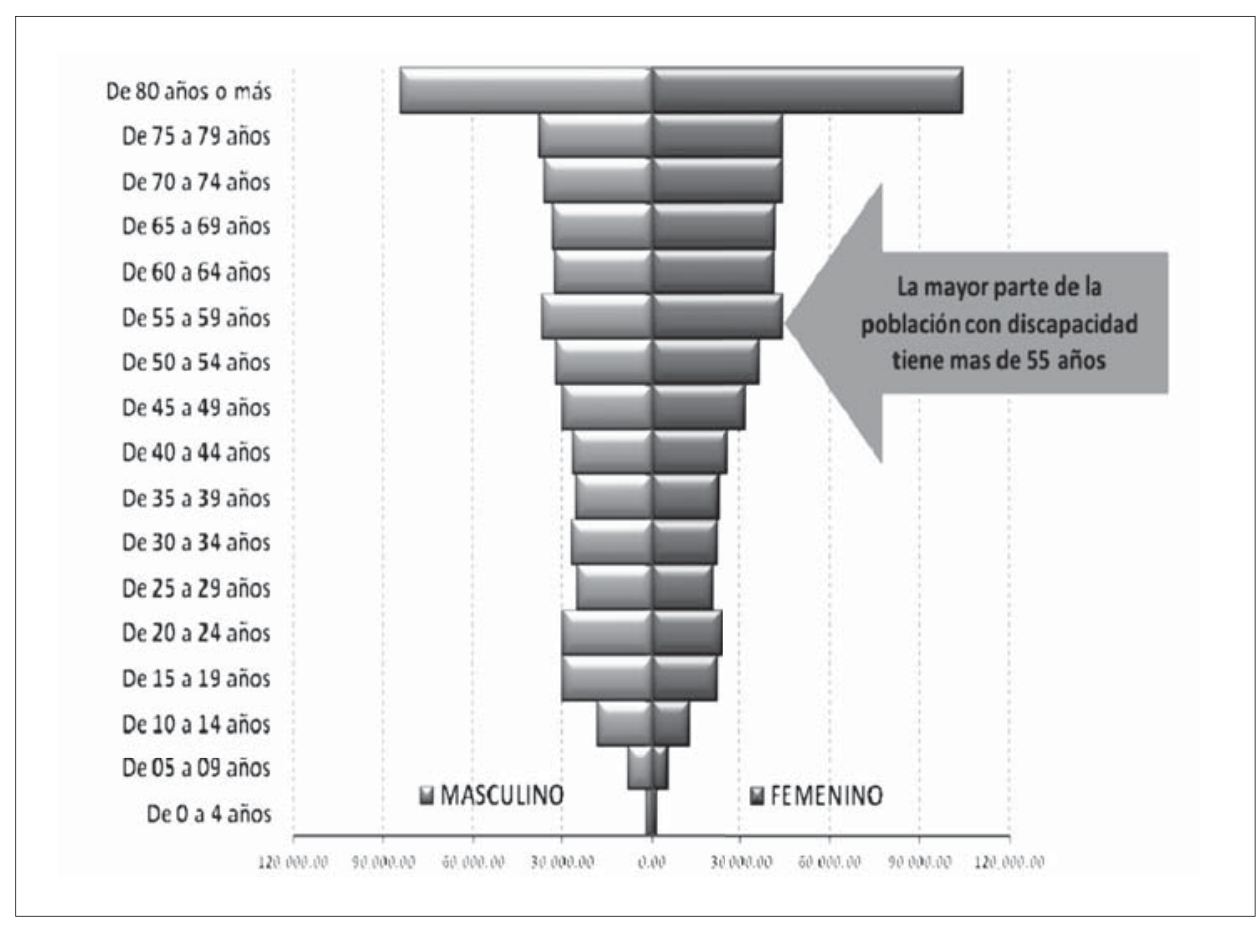

Figura 1. Porcentaje de discapacitados según edad y género proyectados a 2013, a partir del Censo DANE 2005.

Fuente: [2]. 
Otra información importante del Censo 2005 es que la población que tiene alguna discapacidad y se encuentra entre las edades de 5 a 9 años está alrededor del $3 \%$ y el $4 \%$, y los que tienen entre 10 y 14 años están entre el 4\% y el $5 \%$. Por lo tanto, el segmento de población comprendido entre 8 y 15 años es complejo, dado que el desarrollo natural del individuo no permite establecer un patrón de medidas definitivo, lo que lleva a tener una escasez de diseños que permitan una flexibilidad sobre las medidas antropométricas. Por otra parte, los costos de una silla de ruedas son variables debido a factores materiales, funcionales, entre otros. Son pocas las que alcanzan niveles asequibles en precio y que permiten adecuarse fácilmente a una discapacidad en particular.

Respecto al número de limitaciones por persona [2], el 71.2\% presentan una limitación, el $14.5 \%$ dos limitaciones, el $5.7 \%$ tres limitaciones y el $8.7 \%$ tres o más limitaciones permanentes. Del total de personas que reportaron alguna limitación, el 29.3\% poseen limitaciones para moverse o caminar.

Otra estadística interesante fue la que arrojó la caracterización de discapacitados en la ciudad de Bogotá [3], según esta (figura 2), la pirámide poblacional de discapacidad muestra un patrón ascendente con la edad y claramente marcado hacia las mujeres. Asimismo, la mayoría de la población por ciclo vital se encuentra en la etapa de vejez con un

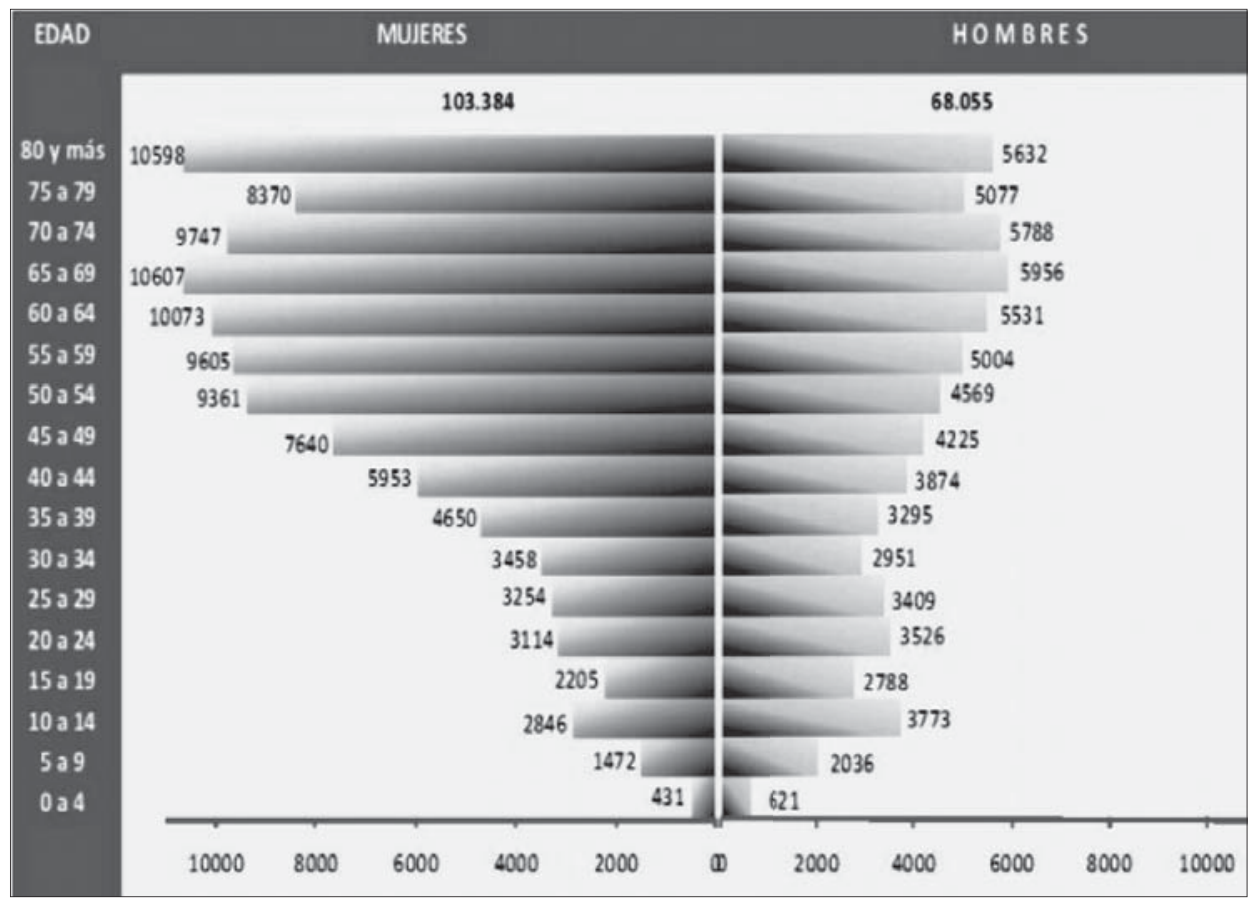

Figura 2. Caracterización de personas con discapacidad en Bogotá. Fuente: [3]. 
$45 \%$, seguida por la adultez con un $40 \%$ y la adolescencia y juventud con un $12.3 \%$. Del total de personas que reportaron alguna limitación, el $28.9 \%$ poseen limitaciones para moverse o caminar. Estas estadísticas corroboran la importancia y potencial impacto que pueden tener, en la calidad de vida de este segmento de la población, los estudios enfocados al diseño y posterior producción de dispositivos que faciliten su movilidad, y que respondan a necesidades específicas.

Existen diversos trabajos de investigación relacionados con el diseño de sillas de ruedas o dispositivos que ayuden a la movilidad de personas discapacitadas, muchos de ellos se basan en la generación de modelos matemáticos para diseñar sistemas de control automático de la misma [5], [6] y [7]. Algunos van un poco más allá, modelando sistemas que le permiten a un paciente estar en un estado erguido [8], otros plantean diseños que puedan detectar obstáculos, girar $360^{\circ}$ o realizar funciones a partir del sensor de voz del propietario como [9], o realizar diseños que ayuden a compensar las limitaciones de personas discapacitadas [10]. En la mayoría de los casos, el diseñador parte del hecho de que los discapacitados requieren de un dispositivo "robótico-avanzado" para poder desarrollarse emocionalmente, cognitivamente y socialmente. Este tipo de suposiciones han hecho que se acoplen diversos elementos al rediseño de una silla de ruedas convencional, lo que ha generado un producto de alto costo al que pocas personas tienen acceso, especialmente en nuestro país donde prevalece una alta proporción de personas con discapacidad pertenecientes a los estratos socioeconómicos y de escolaridad baja (44.6\% estrato I y $35.2 \%$ estrato II).

Otros desarrollos enfocados en la población infantil permiten identificar que la capacidad de movilidad independiente es crucial para un niño en crecimiento y su pérdida puede afectar gravemente el desarrollo cognitivo, emocional y social [11]; sin embargo, y debido a sus altos costos, lo hace inalcanzable a los segmentos sociales más vulnerables, tal como lo expresa [12].

A partir de lo anterior surge la motivación de realizar un estudio sobre una propuesta de diseño de un dispositivo que ayude a las personas con discapacidad motriz entre los 8 y 15 años, con patologías T12 y que no tengan patologías concomitantes en miembros superiores. Un dispositivo que cumpla con las condiciones particulares de los usuarios, incorporando elementos tecnológicos, ergonómicos, legales y prácticos mediante el uso de metodologías de diseño asistido por computador (CAD, Computer Aided Design) y modelamiento por elementos finitos (MEF), entre otras.

Existen diferentes metodologías de diseño (diseño axiomático, método funcional, diseño descriptivo, métodos creativos, método TRIZ, etc.). En este proyecto piloto se ha seleccionado el Método de Función de Calidad (QFD, por sus siglas en inglés) por la gran efectividad que este provee debido a la reducción de fallas que se dan después de la elección final. Lo anterior, con relación a los costos es realmente significativo, pues permite planificar todo proceso tomando como referencia las necesidades de los usuarios o "voz del cliente", esperando satisfacer sus requerimientos en totalidad, y, a su vez, brindando 
resultados de calidad [4]. El QFD es una metodología que ayuda al desarrollo de los procesos de innovación y al desarrollo en las organizaciones, también permite articular la transición que existe entre la generación de una idea y cómo llevarla a cabo. Dicha metodología facilita la determinación de los elementos que la organización necesita para la elaboración de un producto, lo que permite, posteriormente, establecer los métodos de producción para la optimización de los recursos y maximización de los beneficios.

\section{Generalidades del Quality Function Deployment}

El QFD se desarrolló al final de la década de los años sesenta, paralelo a la introducción de productos japoneses originales. Desde sus inicios, fue considerado parte del conjunto de la gestión total de la calidad, conocido en aquel país como Total Quality Control (TQC), y fue diseñado, específicamente, para la creación de nuevas aplicaciones y productos.

\subsection{Estructura del Quality Function Deployment}

La analogía más usada para explicar cómo está estructurado el QFD es una casa de calidad (bouse of quality) [13]. La figura 3 muestra cómo se estructura este tipo de matriz QFD básica. El costado izquierdo, componente 1, es la entrada del cliente; esta es la etapa del proceso en la que se determinan los requerimientos del usuario en relación con el producto (qués). Para satisfacer estas necesidades, se evalúan ciertas especificaciones de desempeño, conformando así el techo interior de la casa o componente 2 (cómos). Posteriormente, se analiza la relación existente entre estos aspectos técnicos, conformándose el techo de la casa (matriz de correlaciones) o componente 3. Asimismo, se debe establecer la relación entre cada una de las necesidades referidas por el usuario con las características técnicas determinadas; esto presentado como componente 4 (matriz de relaciones entre cómos y qués) y para el cual se concede un valor numérico que represente dicha relación.

El fondo o base de la casa, componente 5 (objetivos de los cómos), es donde se jerarquizan los requisitos técnicos del proceso que son críticos. El componente 6 (cómo nos ven), a la derecha, pondera la satisfacción de las necesidades de los usuarios a partir de las propuestas presentadas por la competencia. El componente 7 hace referencia a la relación entre las propuestas disponibles (tanto las de la competencia como la propia) y los componentes técnicos. Finalmente, el componente 8 (evaluación consecución de los objetivos cómos) arroja la propuesta más cercana a satisfacer los requerimientos técnicos.

\subsection{El proceso del Quality Function Deployment}

Un ciclo completo del proceso del QFD lo constituyen seis matrices ilustradas en la figura 3. La matriz 1 se usa para comparar los requerimientos del cliente con las características técnicas del producto. Todas las otras matrices se originan de esta primera 


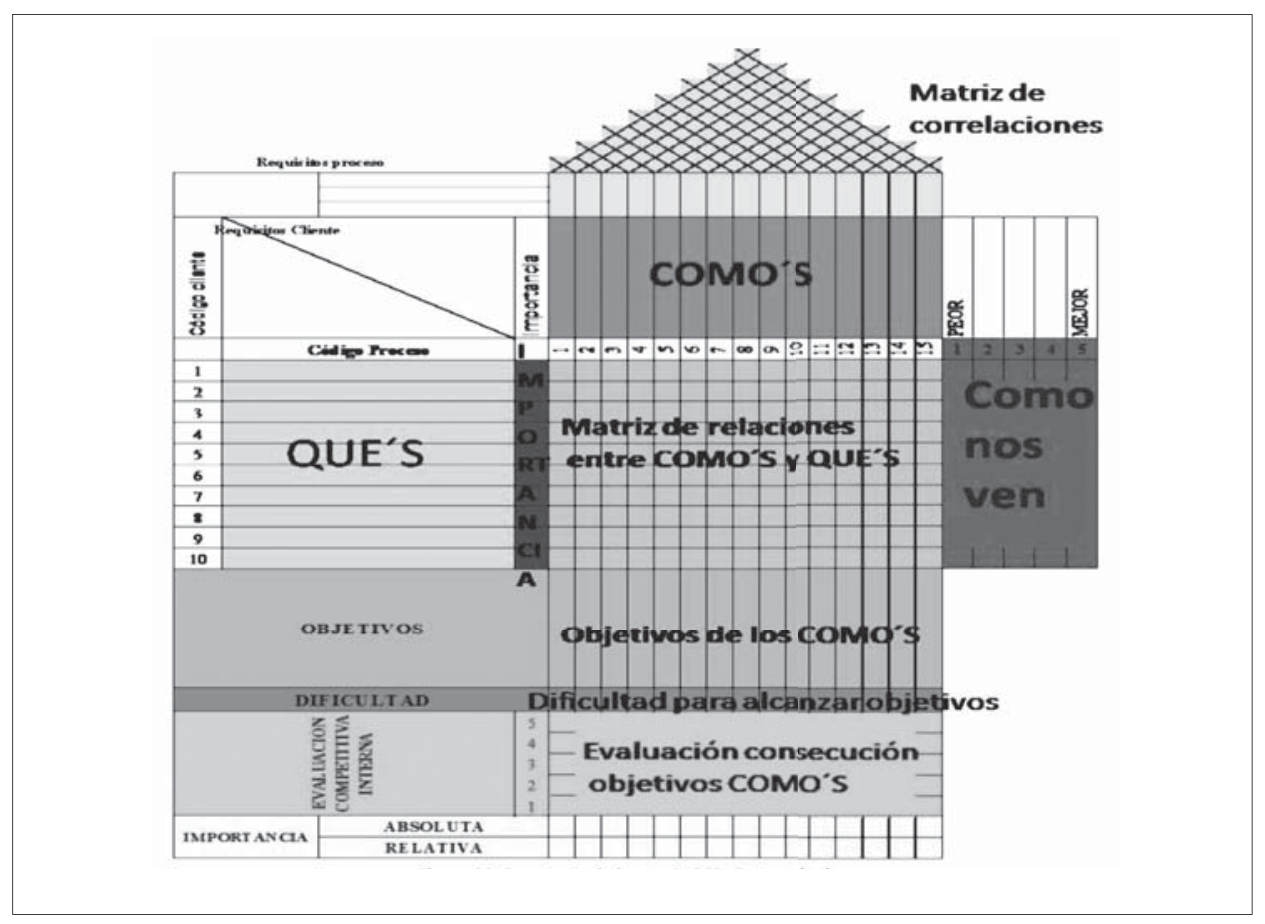

Figura 3. Estructura de la matriz QFD.

Fuente: [14].

matriz. La matriz 2 se usa para comparar las características técnicas en la matriz 1 con sus tecnologías aplicadas asociadas. Sirven para contestar preguntas como: ¿qué quiere el cliente? o ¿cuáles son los requisitos técnicos relacionados con las características que quiere el cliente? La matriz 3 se usa para comparar las tecnologías aplicadas de la matriz 2 con sus procesos de manufactura asociados. La matriz 4 se usa para comparar los procesos de manufactura de la matriz 3 con sus procesos de control de calidad asociados. La matriz 5 se usa para comparar los procesos de control de calidad con sus procesos de control estadístico del proceso. La matriz 6 se usa para comparar los parámetros del control estadístico del proceso con las especificaciones que se han desarrollado para el producto terminado.

\section{Metodología}

\subsection{Captación de las demandas del cliente (voz del cliente)}

Con el fin de captar los requerimientos del cliente ( $\mathrm{RC}$ ), se realizaron encuestas, dado que es un método muy fácil de trabajar y cuyas fuentes primarias son la comunicación con las personas directamente afectadas (patologías de lesión medular de T12 hacia abajo y que no tengan patologías concomitantes en miembros superiores) [15], los centros de aten- 
ción para discapacitados, las familias de los discapacitados, los centros de investigación y el conocimiento propio. Para desarrollar la primera matriz, fue necesario contar con información de cuatro factores:

- Las necesidades reales de los usuarios.

- Cómo valoran los usuarios cada una de las demandas.

- Cuáles son las principales quejas que plantean los usuarios acerca del producto.

- Qué opinión tienen los usuarios de nuestro producto y de los competidores líderes, tomando como referencia cada una de las necesidades detectadas.

A partir de las fuentes mencionadas, se obtuvieron 120 RC. Para poder manejar las necesidades del cliente, estas desben estar estructuradas en jerarquías. Las necesidades primarias son en general las primeras 5 a 10 necesidades que fijan el rumbo estratégico del producto. Por ejemplo, "que sea cómoda" es una necesidad primaria para el dispositivo de transporte. Las necesidades secundarias se elaboran a partir de las necesidades primarias, cada necesidad primaria genera, aproximadamente, entre 3 y 10 necesidades secundarias [16]. La tabla I muestra algunas de ellas, a manera de ilustración.

\begin{tabular}{|c|c|c|}
\hline \multicolumn{3}{|c|}{ Tabla I. Estructuración de las demandas del cliente } \\
\hline Demanda primaria & Demanda secundaria & Demanda terciaria \\
\hline \multirow{5}{*}{ 1. Movilidad } & \multirow{3}{*}{ 1.1. Manejo } & 1.1.1. Autónomo \\
\hline & & 1.1.4. Suave \\
\hline & & 1.1.5. Acceso a unidades sanitarias \\
\hline & \multirow{2}{*}{ 1.3. Fuentes de potencia } & 1.2.1. Humana \\
\hline & & 1.2.2. Eléctrica \\
\hline \multirow{3}{*}{ 4. Antropometría } & 4.1. Dimensiones & 4.1.1. Ajustable \\
\hline & \multirow{2}{*}{ 4.4. Accesorios } & 4.2.1. Apoyos corporales \\
\hline & & 4.2.2. Asiento cómodo \\
\hline \multirow{3}{*}{ 5. Estética } & \multirow{3}{*}{ 5.1. Colores } & 5.1.1. Vivos \\
\hline & & 5.1.2. Conservadores \\
\hline & & 5.2.3. Innovadora \\
\hline \multirow{7}{*}{ 6. Diseño } & \multirow{4}{*}{ 7.1. Calidad } & 7.1.1. Diversos materiales \\
\hline & & 7.1.2. Fácil de ensamblar \\
\hline & & 7.2.4. Simple \\
\hline & & 7.2.6. Facilidad en el giro \\
\hline & 7.3. Medidas & 7.3.1. Comodidad \\
\hline & 7.6 Clima & 7.6.1. Protección contra la lluvia, sol, viento, granizo \\
\hline & 7.7. Seguridad & 7.7.1. Arneses \\
\hline
\end{tabular}

Fuente: los autores. 


\subsection{Priorización de los requisitos del cliente}

No todos los requerimientos del cliente (RC) son igualmente importantes a los ojos de los usuarios; se utilizó el método de análisis jerárquico también conocido como AHP (Analytic Hierarchy Process) que se basa en comparación por pares, para determinar correctamente la importancia de cada demanda. En este caso, los técnicos y usuarios utilizan una valoración del 1 al 5 , donde 5 indica la mayor prioridad y 1 la menor.

La prioridad es uno de los factores que contribuyen al cálculo de la ponderación total de cada característica técnica (CT); el otro factor es la intensidad de la relación RC y CT. Cada pregunta evaluada se realizó teniendo en cuenta la valoración que se muestra en la figura 4.

\subsection{Evaluación del cliente}

La población objetivo evalúa los criterios obtenidos con el fin de definir su grado de satisfacción y determinar su prioridad; estos criterios se comunican a través de preguntas consignadas en una encuesta (figura 4).

Según [17], entrevistar unos 20 o 30 usuarios sería suficiente para identificar el $90 \%$ o más de las necesidades en un segmento relativamente homogéneo. Por lo

\begin{tabular}{|c|c|c|}
\hline & ENCUESTA & $\mathbf{N}^{0}$ \\
\hline \multicolumn{3}{|c|}{ Nombre: } \\
\hline \multicolumn{3}{|c|}{ Edad: __ Genero: __ Tiempo que lleva con la limitación: } \\
\hline \multicolumn{3}{|c|}{ Tipo de lesión: } \\
\hline \multicolumn{3}{|c|}{ Forma actual de movilización: } \\
\hline \multicolumn{3}{|c|}{$\begin{array}{l}\text { La siguiente encuesta busca obtener las caracteristicas que son consideradas muy importantes al momento de } \\
\text { generar el desarrollo de un dispositivo de movilidad para personas discapacitadas. } \\
\text { Para el desarrollo de la encuesta debe tener en cuenta: 1) leer atentamente la pregunta. 2) al momento de } \\
\text { realizar la valoración su significado es el siguiente (5) Necesario, (4) Muy importante, (3) Importante, (2) Poco } \\
\text { importante, (1) No es necesario. }\end{array}$} \\
\hline & ¿Considera importante la autonomía en la movilidad? & Valoración: (5)(4)(3)(2)(1) \\
\hline & $\begin{array}{l}\text { ¿Qué tan importante es la colaboración de una personaal momento de } \\
\text { movilizarse? }\end{array}$ & Valoración: (5)(4)(3)(2)(1) \\
\hline & $\begin{array}{l}\text { ¿Qué tan relevante es la posible ayuda de otra persona al momento de } \\
\text { movilizarse? }\end{array}$ & Valoración: (5)(4)(3)(2)(1) \\
\hline & ¿Considera que el movimiento del dispositivo debe ser suave? & Valoración: (5)(4)(3)(2)(1) \\
\hline & ¿Desea que pueda tener acceso a las unidades sanitarias estándar? & Valoración: (5)(4)(3)(2)(1) \\
\hline & ¿Cuál es su criterio frente al dispositivo se mueva por su propia cuenta? & Valoración: (5)(4)(3)(2)(1) \\
\hline & $\begin{array}{l}\text { ¿Si el dispositivo tuviera controles para conducirlo que tan importante es } \\
\text { que serán pequeños? }\end{array}$ & Valoración: (5)(4)(3)(2)(1) \\
\hline
\end{tabular}

Figura 4. Encuesta elaborada en el estudio. Fuente: los autores. 
tanto, posterior a la aplicación de la encuesta, se realiza la tabulación de los datos por medio de un análisis matricial realizado con la ayuda del software de Excel 2007® de Microsoft. El análisis arrojó que existían 41 necesidades primarias: autonomía, colaboración para movilizarse, movimiento suave, acceso a unidades sanitarias, energía humana, liviano, ruedas medianas para la dirección, frenos mecánicos, posición sentada, entre otras.

\subsection{Elaboración de la lista de parámetros técnicos o características técnicas}

Las CT también pueden organizarse en un árbol jerárquico. Por medio del método tormenta de ideas se obtienen los elementos que se enuncian a continuación: peso completo, peso del chasis, altura, ancho, longitud, diámetro de ruedas delanteras, altura al asiento, alto del espaldar, entre otros.

\subsection{Ponderación, evaluación y correlación}

\subsubsection{Relaciones entre requerimientos del cliente y características técnicas}

En el cuadro central de la matriz se asignó un valor que informará el nivel de importancia que tiene la relación entre las filas de los RC y las columnas de las CT. Esta correlación expresa cuánto afecta a cada RC una CT particular.

- Primer criterio: débil, afirma que la relación encontrada entre las características técnicas y requisitos del cliente resta de tener gran importancia. Se le asigna valor de 1.

- Segundo criterio: moderada, se asigna este criterio cuando existe una relación entre las variables. Se le asigna valor de 3.

- Tercer criterio: fuerte, que quiere representar la existencia de una alta dependencia o relación entre los criterios que se desean analizar. Se le asigna valor de 9.

\subsubsection{Relación entre características técnicas}

La relación entre características técnicas se describe brevemente a continuación:

- Primer criterio: es muy fuerte, y lo relaciona con el símbolo “++", que significa que la relación de las características técnicas tiene un nivel de dependencia muy alto.

- Segundo criterio: es fuerte, y lo relaciona con el símbolo “+”, esta relación es de dependencia, pero no muy fuerte.

- Tercer criterio: es nada, no se ha determinado el grado de relación. 
- Cuarto criterio: es débil, y se simboliza “-”, que quiere decir que el criterio relacionado tiene un nivel de independencia bajo.

- Quinto criterio: es muy débil, y se le agina el símbolo de “- -", que quiere representar un alto nivel de independencia.

\subsection{Nivel de dificultad}

Existen 5 niveles que determinarán cuál es el nivel de dificultad que existe para los usuarios. Inicia con el criterio de "muy fácil" con un valor asignado de 1 hasta una ponderación de "muy complicado" con un valor de 5.

\subsection{Puntuación absoluta}

Es el valor que se genera al momento de evaluar las características técnicas en relación con los requerimientos del cliente es llamada la puntuación absoluta esta puntuación se relaciona con el nivel de dificultad asignado a cada una de las características técnicas. La fórmula para calcularla es la siguiente:

Puntuación absoluta $=\sum_{0}^{\mathrm{j}}$ relaciones entre RT y CT $\times \sum_{0}^{\mathrm{i}}$ Calificación de la importancia del RT

X Nivel de dificultad de cada una de las $C T_{j}$

Donde i es la cantidad de requisitos del cliente y j es la cantidad de características técnicas.

\subsection{Puntuación relativa}

Se refiere al modo con el cual se calcula el posicionamiento de la puntuación absoluta de una característica técnica con la puntuación absoluta de la cantidad de características técnicas analizadas. La fórmula para calcularla es la siguiente:

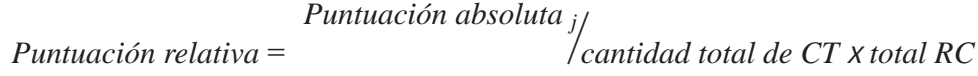

\section{Resultados y discusión de los resultados}

\subsection{Primera casa de la calidad}

El primer resultado obtenido es la primera casa de la calidad, la cual se muestra en la figura 5. En ella se describen las CT que más influyen o pueden solucionar los RC. Esta primera matriz arrojó como resultado $36 \mathrm{CT}$. 


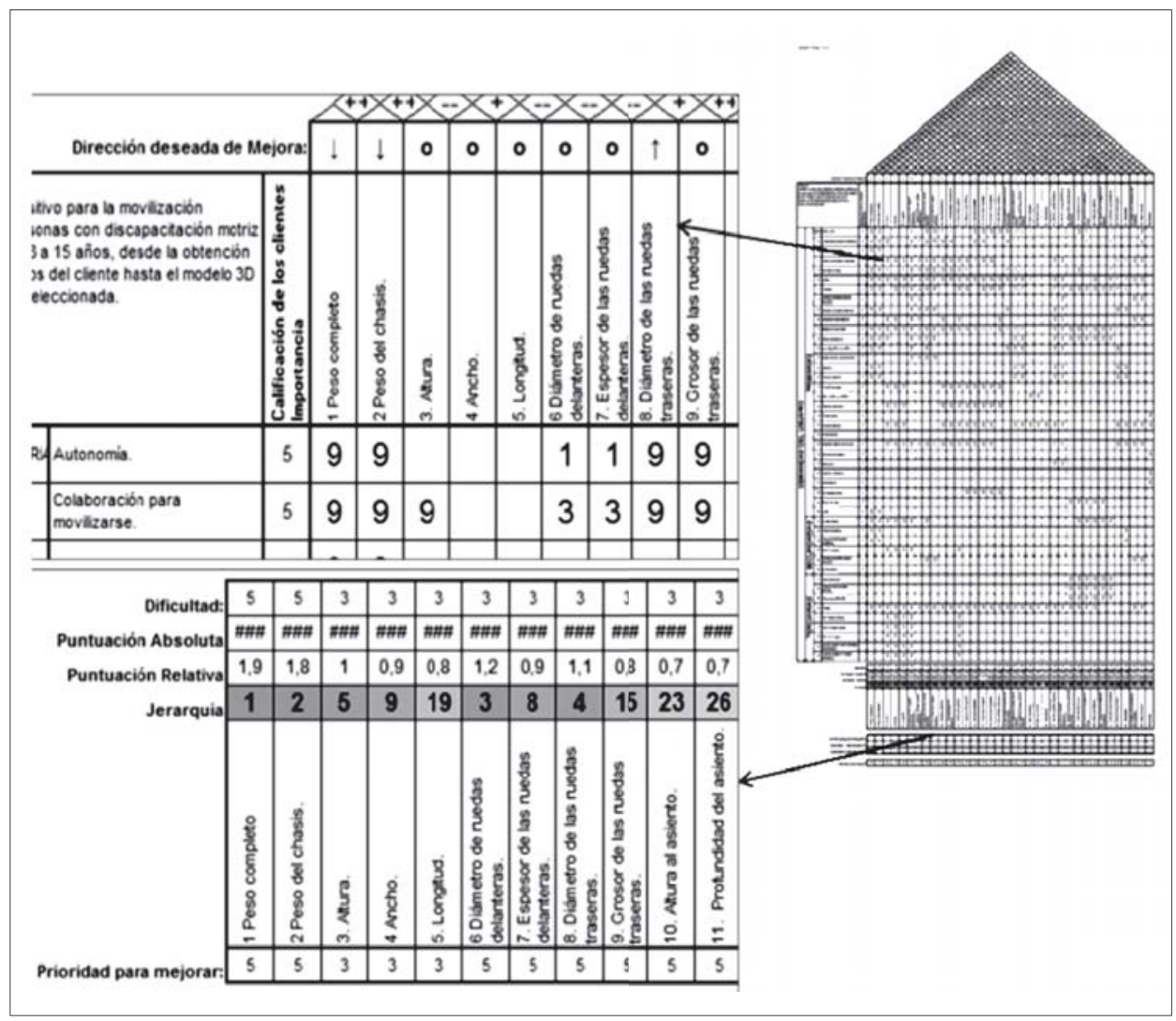

Figura 5. Matriz 1 QFD.

Fuente: Ios autores.

\subsection{Análisis Morfológico}

El Análisis Morfológico es un método analítico-combinatorio cuyo objetivo es resolver problemas mediante el análisis de las partes que lo componen. Se basa en la concepción de que cualquier objeto de nuestro pensamiento está compuesto o integrado por un cierto número de elementos, los cuales tienen identidad propia y pueden ser aislados.

Para este fin se siguieron las recomendaciones dadas en [18] para determinar con éxito las características que hacen parte del análisis morfológico, a saber:

- Generalidad e independencia entre características o funciones. Aquí se evaluó la dependencia, independencia o no validez de la característica para tenerlas en cuenta, agruparlas y realizar los diseños. En la tabla II se ilustran algunas.

- Características o funciones factibles.

- Una lista razonable y manejable debe tener de 4 a 8 características o funciones. 


\begin{tabular}{|c|c|c|c|c|c|}
\hline \multicolumn{6}{|c|}{ Tabla II. Relacionamiento de las características } \\
\hline CT & 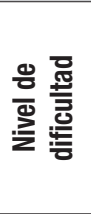 & 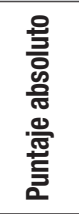 & 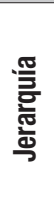 & 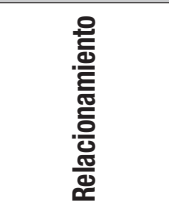 & Explicación \\
\hline $\begin{array}{l}\text { 01. Peso } \\
\text { completo }\end{array}$ & 5 & 3050 & 1 & Dependiente & $\begin{array}{l}\text { Se unificará en un criterio llamado AUTONO- } \\
\text { MÍA, su relación con el peso está dada en que } \\
\text { a mayor peso menor es la posibilidad de tener } \\
\text { autonomía. }\end{array}$ \\
\hline $\begin{array}{l}\text { 06. Diámetro } \\
\text { de ruedas } \\
\text { delanteras }\end{array}$ & 3 & 1901 & 3 & Independiente & Se analizará en la segunda casa de la calidad. \\
\hline 33. Engranaje & 5 & 1350 & 16 & No válido & $\begin{array}{l}\text { La característica no se tendrá en cuenta al es- } \\
\text { tar en contra de la autonomía y de la genera- } \\
\text { ción de peso al diseño. }\end{array}$ \\
\hline $\begin{array}{l}\text { 34. Grados } \\
\text { inclinación } \\
\text { espaldar }\end{array}$ & 3 & 758 & 31 & Válido & $\begin{array}{l}\text { La silla debe poseer una manera que facilite la } \\
\text { inclinación de la silla. }\end{array}$ \\
\hline 35. Radio de giro & 2 & 747 & 32 & Independiente & $\begin{array}{l}\text { Es una característica obligatoria, el dispositivo } \\
\text { debe girar } 360^{\circ} \text {. }\end{array}$ \\
\hline 36. Pintura & 1 & 230 & 35 & Independiente & $\begin{array}{l}\text { Es una característica que en el diseño no gene- } \\
\text { ra un factor de preponderancia. }\end{array}$ \\
\hline
\end{tabular}

Fuente: Ios autores.

La tabla III ilustra un resumen de las características necesarias para realizar las propuestas de diseño. Se agruparon de la siguiente manera: autonomía, energía de propulsión, ruedas, inclinación de las ruedas, amortiguación, cambios climáticos y comodidad.

Tabla III. Características morfológicas

\begin{tabular}{|l|l|}
\hline $\begin{array}{l}\text { Característica } \\
\text { Morfológica }\end{array}$ & \multicolumn{1}{c|}{ Explicación } \\
\hline Autonomía & $\begin{array}{l}\text { La autonomía es un criterio morfológico que se desprende a partir de las entrevistas realizadas } \\
\text { a los usuarios, en donde ellos describen que lo más importante es que ellos sean capaces de } \\
\text { realizar los movimientos del dispositivo por sí mismos, dada esta razón resulta ser una de las } \\
\text { más críticas en la realización del diseño. }\end{array}$ \\
\hline $\begin{array}{l}\text { Energía de } \\
\text { propulsión }\end{array}$ & $\begin{array}{l}\text { Existen diferentes opciones de propulsión (mecánica, eléctrica, neumática, humana) que po- } \\
\text { sibilitan la movilidad de este dispositivo. Se le da mayor importancia a la energía humana ya } \\
\text { que, según las encuestas, las personas discapacitadas se sienten rechazadas por la sociedad } \\
\text { por su condición y al hacer un dispositivo que no intervengan sus brazos como mecanismo de } \\
\text { propulsión se sentirían aún peor anímicamente. Otra razón es que es el único medio por el cual } \\
\text { ellos realizan una actividad física, y además no genera mayor peso como otros dispositivos. }\end{array}$ \\
\hline
\end{tabular}




\begin{tabular}{|l|l|}
\hline Ruedas & $\begin{array}{l}\text { Existe una diversidad en la cantidad de ruedas que se pueden definir en el dispositivo, en este } \\
\text { caso según los requisitos obtenidos por parte del cliente está dada por } 3,4 \text { y } 5 \text { ruedas; } 3 \text { ruedas } \\
\text { por ser un sistema más moderno, } 4 \text { ruedas por ser un sistema tradicional y } 5 \text { ruedas para mayor } \\
\text { estabilidad. }\end{array}$ \\
\hline $\begin{array}{l}\text { Inclinación } \\
\text { de las ruedas }\end{array}$ & $\begin{array}{l}\text { La inclinación de las ruedas responde a que si tienen una inclinación de } 5 \text { grados hacia adentro } \\
\text { le permitirá realizar desplazamientos más estables, pero aumenta el ancho del dispositivo. Tener } \\
\text { las ruedas en un estado vertical permitirá tener desplazamientos menos estables pero el ancho } \\
\text { del dispositivo es menor. }\end{array}$ \\
\hline Amortiguación & $\begin{array}{l}\text { Se establecen dos tipos de amortiguación, a partir de un pistón neumático y otro hidráulico, } \\
\text { como opciones que favorecen un desplazamiento estable y suave. }\end{array}$ \\
\hline $\begin{array}{l}\text { Cambios } \\
\text { climáticos }\end{array}$ & $\begin{array}{l}\text { Dentro las decisiones consideradas, se destaca la de tener la posibilidad de que el dispositivo } \\
\text { tenga protección climática. }\end{array}$ \\
\hline Comodidad & $\begin{array}{l}\text { La comodidad es un elemento de suma importancia, al momento de realizar las encuestas, se } \\
\text { encontró que es relevante dado que sin esta cualidad puede ocasionar efectos adversos en la } \\
\text { salud de las personas, como la generación de llagas, o malformaciones de las extremidades. }\end{array}$ \\
\hline
\end{tabular}

Fuente: los autores.

\subsubsection{Bocetos de dispositivos móviles}

Estos bocetos fueron realizados por medio del software Solid Edge, tomando como partida las características técnicas obtenidas de la primera casa de calidad y que, posteriormente, fueron reducidas por medio del método de análisis morfológico. Las diferentes características de análisis morfológico y algunas opciones de diseño se muestran en la tabla IV.

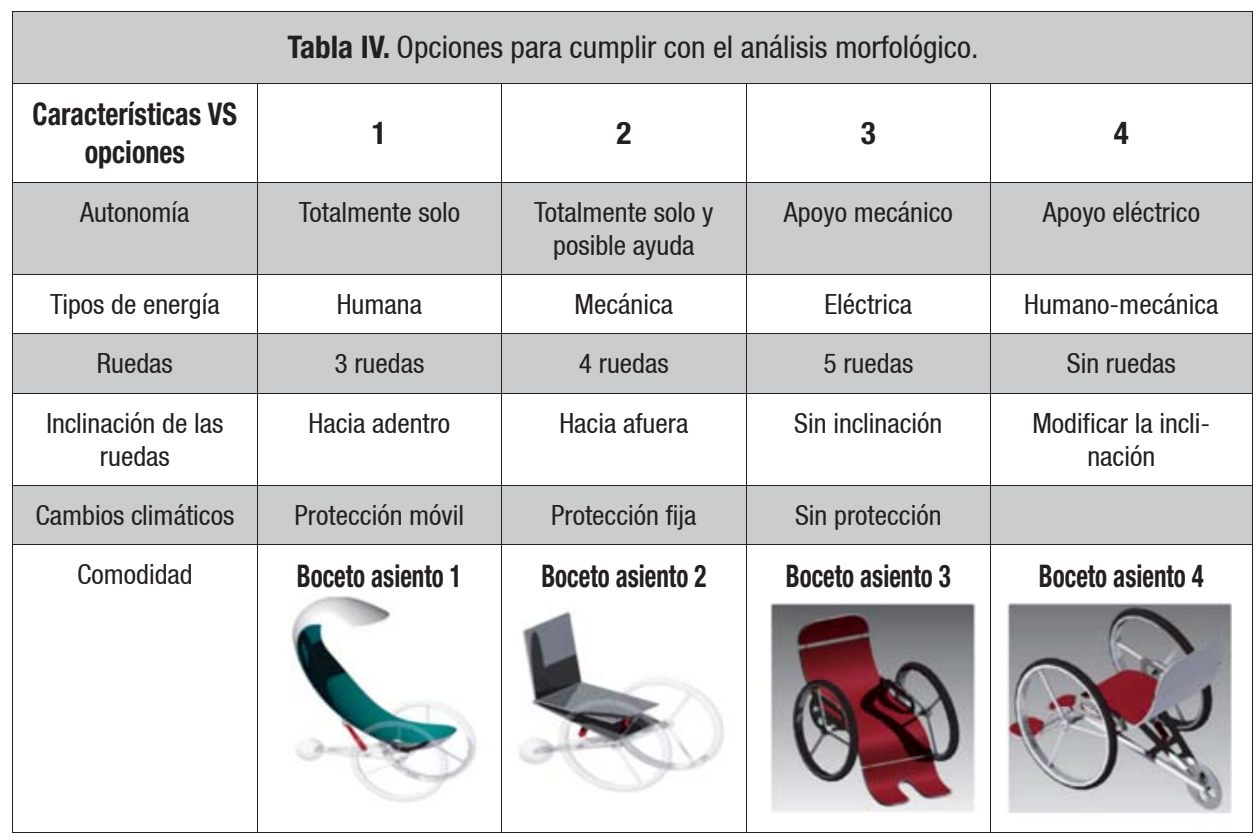

Fuente: Ios autores. 


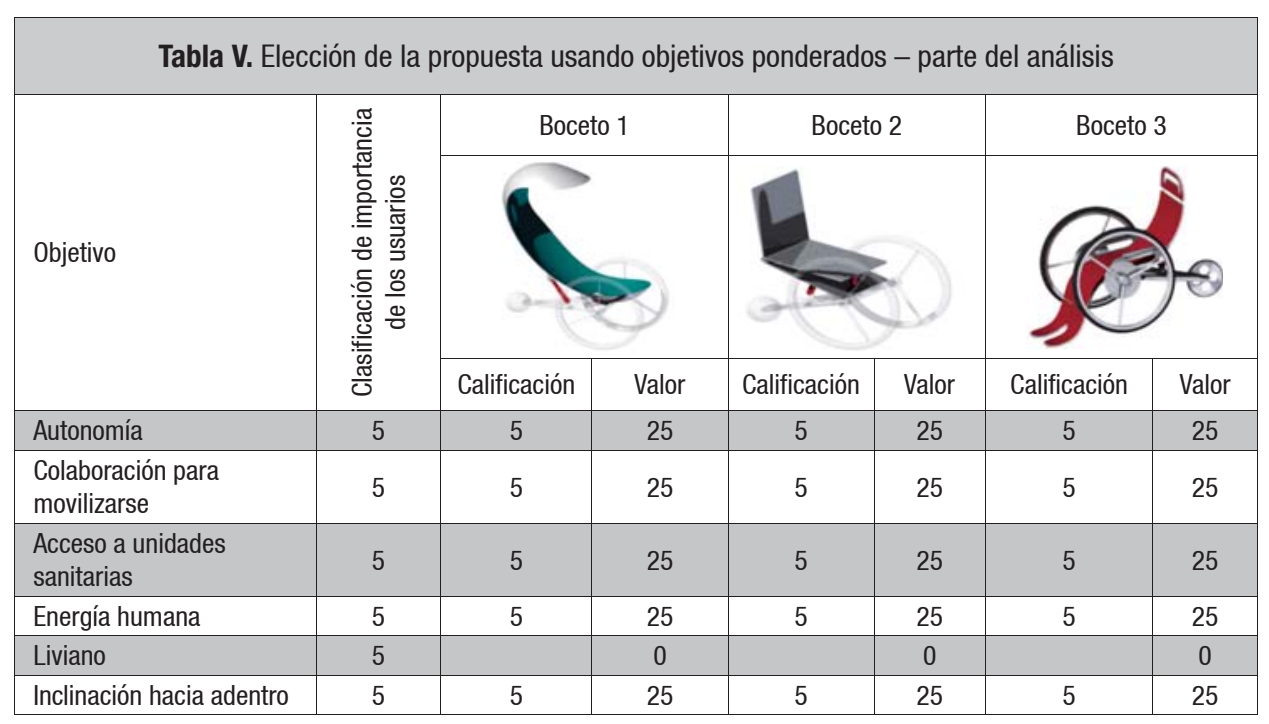

Fuente: los autores.

Para la elección de la mejor propuesta se realiza un análisis de objetivos ponderados [19]. Para ello, se construye la matriz de criterios y valoración (parcialmente ilustrada en la tabla V). En esta matriz el diseñador es quien asigna la calificación, de tal manera que será de 1 cuando su cumplimiento del objetivo sea bajo, 3 cuando lo cumple en manera parcial y 5 cuando los cumple totalmente. Se observa como resultado de este análisis que la propuesta 1 es la que tiene mayor cumplimiento de los requisitos del cliente. Seguidamente, para obtener el valor del objetivo, el diseñador deberá multiplicar su calificación con la calificación de la importancia para los usuarios, que es obtenida a partir de la primera matriz de la casa de la calidad.

Algunas razones por las cuales fueron o no seleccionas son: la opción 2 no es seleccionada por cuanto la forma del asiento dificulta convertirla en un sistema plegable o ajustable. La opción 3 es un asiento que se parece a una silla hospitalaria; para los encuestados no es agradable la forma de la silla puesto que a algunos les trae malos recuerdos de su discapacidad. La opción 4 no es seleccionada ya que posee un tipo de asiento y espaldar no adaptable a los usuarios en periodo de crecimiento (entre 8 y 15 años de edad). Así entonces, la propuesta 1 es la que tiene mayor cumplimiento de los RC.

\subsection{Propuesta seleccionada}

A continuación, se muestran brevemente aspectos relevantes de la propuesta seleccionada (boceto 1). Para la selección de los materiales adecuados (tabla VI), se tuvo en cuenta la segunda casa de calidad QFD (no mostrada en este artículo). 


\begin{tabular}{|c|l|l|c|}
\hline \multicolumn{4}{|c|}{ Tabla VI. Materiales producto de segunda casa de calidad QFD } \\
\hline Número de elemento & \multicolumn{1}{|c|}{ Título } & \multicolumn{1}{c|}{ Material } & Cantidad \\
\hline $1^{*}$ & Base principal & Polipropileno, alto impacto & 1 \\
\hline $2^{*}$ & Soporte estructural & Aluminio 1060, plancha estriada & 1 \\
\hline $3^{*}$ & Pivote & Acero & 2 \\
\hline 4 & Rueda de empuje & Aluminio, 1060 & 2 \\
\hline 5 & Rueda dirección & PVC & 1 \\
\hline 6 & Base amortiguador & Acero & 1 \\
\hline $7^{*}$ & Alma del amortiguador & Acero & 1 \\
\hline $8^{*}$ & Cabecera & Polipropileno, alto impacto & 1 \\
\hline $9^{*}$ & Apoya pies & Polipropileno, alto impacto & 1 \\
\hline $10^{*}$ & Freno & Titanio, sin aleación & 2 \\
\hline 11 & Protector & Polipropileno, propósito general & 1 \\
\hline
\end{tabular}

Fuente: Ios autores.

Algunos elementos adicionales en la silla son el apoya brazos, esencial para el diseño como parte de los elementos de comodidad que necesitan en el dispositivo, el sistema de amortiguación, los frenos de disco, el apoyo en todo el cuerpo de la persona, el apoya pies y apoya cabeza con la opción de extenderlos ya que, como se mencionó, las personas están en periodo de crecimiento (figura 6).

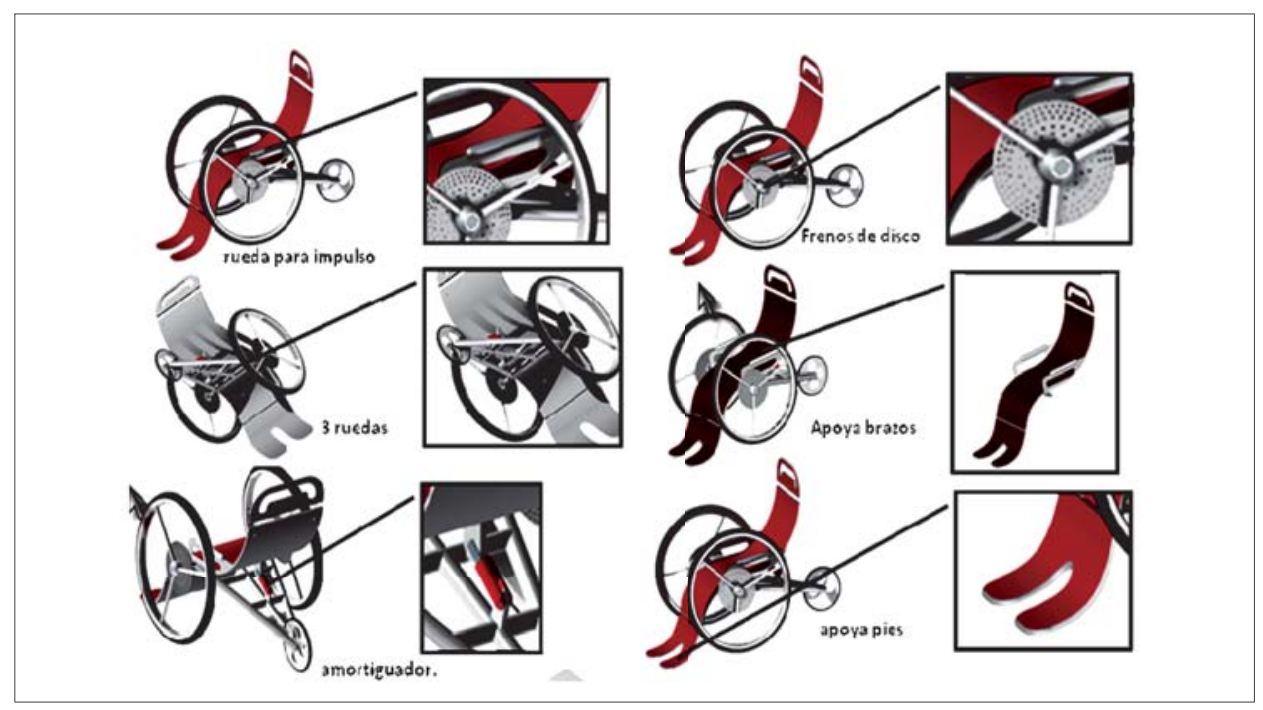

Figura 6. Detalles del diseño seleccionado Fuente: los autores. 


\section{Conclusiones}

Una de las ventajas que se observó durante la aplicación de la metodología de diseño para el producto de movilidad, es que permite la eliminación de la subjetividad a lo largo de su proceso. Lo anterior, se logra gracias a técnicas como el análisis morfológico para la toma de decisión del boceto elegido y el análisis de objetivos ponderados para determinar el nivel de relación que existe entre las características que se evalúan en las matrices de la casa de la calidad.

Por otra parte, durante el proceso de levantamiento de $\mathrm{RC}$, se encontraron varios de carácter muy críticos que solamente los usuarios o población objetivo (las personas con discapacidad) podían conocer, por ejemplo, que el dispositivo sea liviano debido a que las extremidades inferiores son las diseñadas para resistir el peso de las personas, los brazos no están diseñados para dicha labor. Por lo tanto, si se exigen mucho los músculos de los brazos estos pueden desarrollar lesiones.

Igualmente, el estudio identificó que los usuarios de sillas de ruedas encuestados no se encuentran totalmente satisfechos con ellas. Al respecto, la encuesta que fue procesada durante el proceso QFD ayudó a captar con mayor facilidad los requisitos que verdaderamente son importantes para crear un boceto de diseño que puede mejorar las condiciones de comodidad de los usuarios y, en general, las condiciones de vida de niños $\mathrm{y}$ adolescentes que se encuentran en periodo de crecimiento.

La propuesta descrita en este artículo está basada explícitamente en las necesidades de las personas discapacitadas. En efecto, tras el desarrollo de la casa de la calidad se generaron prototipos que no se alejan de la forma básica con que se cuenta actualmente, teniendo en cuenta los requerimientos antropométricos, los aspectos ergonómicos, entre otros. En etapas posteriores asociadas a este proyecto se realizará el análisis estático y dinámico de la estructura del dispositivo, así como el análisis de todas sus partes constitutivas y optimización de dimensiones y material mediante software de elementos finitos. Después de ello se identificarán los procesos de fabricación de una silla piloto. De esta manera, se espera en el futuro lograr construir un dispositivo que cumpla con los requisitos de cliente y criterios técnicos arrojados por el QFD, pero con materiales económicos y procesos de fabricación que conlleven a una silla de un bajo costo.

\section{Referencias bibliográficas}

[1] Organización Mundial de la Salud y Banco Mundial, "Resumen informe mundial sobre la discapacidad," [Online]. Disponible en: http://www.who.int/disabilities/world_report/2011/summary_es.pdf. Consultado: septiembre 15 de 2012.

[2] Ministerio de Salud y Protecciòn Social (MinSalud), "Sistema Nacional de Discapacidad (SND)," [Online]. Disponible en: http://www.minsalud.gov.co/proteccionsocial/Paginas/DisCAPACIDAD.aspx. Consultado: junio 16 de 2014. 
[3] A. González. Actualizacion de la caracterización sociodemografica de la población con discapacidad en bogotá; 2005-2010. Bogotá: Secretaria Distrital de Salud, 2011.

[4] J. Acuña. Mejoramiento de la calidad, un enfoque a los servicios. Costa Rica: Editorial Tecnológica de Costa Rica, 2005.

[5] S. Ahmad, N. Siddique and M. Tokhi, "Modelling and Simulation of Double-Link Scenario in a Two-Wheeled Wheelchair," Integrated Computer-Aided Engineering, vol. 21, no. 2, pp. 119-132, 2014.

[6] C. B. Brutti, A. J. Sattler, D. Albacetti, A. R. Canavelli and C. B. Donisi, "Motorización de sillas de ruedas convencionales: unidad totalmente desarmable y con exclusivo sistema de desacople de motores para tracción manual," Actas de Ingeniería Clínica y Tecnología Médica, vol. 1, no. 15, pp.110-125, 2010.

[7] Crea \& Ajuda S.L. and M. A. Torra-Sorribes, "Wheelchair that can be raised and converted into a stretcher," [Online], Patente No. WO 2009010602 A1, 2013. Disponible en: https://www. google.com/patents/WO2009010602A1?cl=en. Consultado: junio 20 de 2012.

[8] L. Salcedo, C. Torres, G. Urriolagoitia y B. Romero, "Rediseño para la optimización de una silla de ruedas eléctrica de dos posiciones," en $10^{\circ}$ Congreso Nacional de Mecatrónica, Puerto Vallarta (Jalisco), noviembre 3 y 4 de 2011.

[9] A. Navarro, B. Elizalde, D. Camacho y N. Hernandez, "Silla de ruedas súper inteligente," [Online]. Disponible en: http://www.tlalpan.uvmnet.edu/oiid/download/Silla\%20de\%20ruedas\%20inteligentes_04_ING_ISC_PIT_E.pdf. Consultado: julio 10 de 2012.

[10] J. S. Aguasvivas y L. González, "Diseño de refrigerador para personas en silla de ruedas," en The Latin American and Caribbean Conference for Engineering and Technology - LACCEI 2009, San Cristobal (Venezuela), junio 2 de 2009.

[11] L. Lugo y V. Seijas, "La discapacidad en Colombia: una mirada global," Revista Colombiana Medicina Física y Rehabilitación, vol. 22, no. 2, pp. 64-122, 2013.

[12] H. Soh and Y. Demiris, "Involving young children in the design of a safe, smart paediatric wheelchair". Submitted to the ACM/IEEE HRI-2011 Pioneers Workshop, Lausanne, 2011.

[13] J. C. De la Iglesia, F. Martín y E. Yacuzzi, "El método de Kano en el diseño de productos y servicios," [Online]. Disponible en: http://www.ucema.edu.ar/publicaciones/download/documentos/224.pdf. Consultado: julio 10 de 2012.

[14] D. L. Goetsch, "Introduction to Total Quality," in Quality Function Deployment. New Jersey: Merrill, 1997, pp. 245-279.

[15] B. Guillon, "Sillas de ruedas," [Online]. Encyclopédie Médico-Chirurgicale. E - 26-170-B-10. Disponible en: http://www.slideshare.net/normaobaid/sillas-de-ruedas-28671370. Consultado: julio 10 de 2012.

[16] J. Hauser and A. Griffin, "Voice of the customer," Marketing Science, vol. 12, no. 1, pp. 1-27, 1993.

[17] J. Alcaide. Diseño de producto, métodos y técnicas. Valencia: Editorial de la UPV, 2001.

[18] F. Martín y E. Yacuzzi, "Matrices de calidad y diseño de nuevos," Interpharma, año IV, no. 10, pp. 20-24, 2001.

[19] E. Yacuzzi, "La gestión hoshin: un marco para la calidad," Interpharma, año V, no. 12, pp. 4046, 2002. 
Diseño de un dispositivo para la movilidad de personas con discapacidad motriz usando el método función de calidad

\section{Leonardo Emiro Contreras Bravo}

Ingeniero mecánico. Magíster en Ingeniería. Docente de planta de la Universidad Distrital Francisco José de Caldas. Director del grupo de investigación Dimsi (Diseño Modelamiento y Simulación).

Correo electrónico: lecontrerasb@udistrital.edu.co

\section{Wilmar Augusto Granados}

Estudiante de Ingeniería Industrial en la Universidad Distrital Francisco José de Caldas. Miembro del grupo de investigación Dimsi (Diseño Modelamiento y Simulación).

Correo electrónico: wagranados@udistrital.edu.co 